\title{
Treatment Outcomes in Endodontics
}

\author{
${ }^{1}$ Raghu Srinivasan, ${ }^{2}$ Ramya Raghu
}

\section{ABSTRACT}

The success of endodontic treatment has been of great interest to practitioners for many years now. Endodontic failures, which are of particular interest to us, are due to either microbial or nonmicrobial reasons. It is often thought that procedural errors like ledging, perforation, overfilling, underfilling or instrument separation invariably result in failure. In reality, the fact is that such mishaps only impede the accomplishment of an ideal root canal treatment. It is often the concomitant presence of infection that ultimately results in failure.

Numerous studies have evaluated the success or failure of endodontic therapy and reported a wide range of success rates from 40 to $93 \%$. This wide range may be due to differences in clinical procedure, experimental design, criteria for evaluation and the length of the observation period. However, most authors conclude that the crucial factor influencing success is the preoperative status of the tooth. Teeth with an apical radiolucency show a $20 \%$ lower success rate than teeth without lesion.

Till date, the main method of evaluating success is radiographic, using recall radiographs. This method, however, is not without its own limitations due to examiner bias and inconsistencies.

The factors affecting treatment outcome include those related to diagnosis, preoperative condition of the patient and tooth, standard of care during treatment, postoperative factors and ongoing maintenance. This paper reviews the various factors influencing the outcome of endodontic treatment in light of modern thought.

Keywords: Failure, Postoperative factors, Preoperative factors, Standard of care, Success, Treatment outcome.

How to cite this article: Srinivasan R, Raghu R. Treatment Outcomes in Endodontics. J Oper Dent Endod 2016;1(1):13-17.

Source of support: Nil

Conflict of interest: None

\section{INTRODUCTION}

Modern endodontic treatment is highly predictable with a degree of success approaching 100\%. The success

\footnotetext{
${ }^{1,2}$ Professor and Head

${ }^{1}$ Department of Conservative Dentistry, AECS Maaruti College of Dental Sciences and Research Center, Bengaluru, Karnataka India

${ }^{2}$ Department of Conservative Dentistry, Bangalore Institute of Dental Sciences, Bengaluru, Karnataka, India

Corresponding Author: Ramya Raghu, Professor and Head Department of Conservative Dentistry, Bangalore Institute of Dental Sciences, Bengaluru, Karnataka, India, e-mail: ramyadev21@gmail.com
}

of endodontics treatment has been of great interest to practitioners for many years now. The various causes for failure of treatment rendered may be divided into prosthetic, periodontal and endodontic failures. ${ }^{1}$ Prosthetic failures are due to crown fracture, root fracture or failure of the restorations to the extent that the tooth is deemed unrestorable. Periodontal failures relate to extensive bone loss and mobility. ${ }^{1}$ Endodontic failures, which are of particular interest to us are due to either microbial or nonmicrobial reasons. It is often thought that procedural errors like ledging, perforation, overfilling, underfilling or instrument separation invariably result in failure. In reality, the fact is that such mishaps only impede the accomplishment of an ideal root canal treatment. It is often the concomitant presence of infection that ultimately results in failure. ${ }^{2}$

Numerous studies have evaluated the success or failure of endodontic therapy and reported a wide range of success rates from 40 to $93 \% .^{3}$ This wide range may be due to differences in clinical procedure, experimental design, criteria for evaluation and the length of the observation period. However, most authors conclude that the crucial factor influencing success is the preoperative status of the tooth. Teeth with an apical radiolucency show a $20 \%$ lower success rate than teeth without lesion. ${ }^{3}$

Till date, the main method of evaluating success is radiographic, using recall radiographs. This method, however, is not without its own limitations due to examiner bias and inconsistencies. ${ }^{1}$

This paper reviews the various factors influencing the outcome of endodontic treatment in the light of modern thought.

\section{DEFINING SUCCESS AND FAILURE- TRADITIONAL CONCEPTS VS CURRENT THOUGHT}

The so-called 'Strindberg concept' defined the standards for success and failure based on stringent criteria. According to Strindberg (1956), 'success' is a clinically symptom-free tooth with a radiologically normal periapex after a predetermined postoperative period. This implies clinical, radiologic and histologic resolution of inflammation, whereas, 'failure' is the presence of signs or symptoms indicating disease. ${ }^{3}$ This concept is perceived to be too dogmatic or black and white and is considered inflexible for everyday clinical use. 
To strike a rational middle path, considering patient comfort and function on one hand and reasonable evidence of healing on the other, the following guidelines proposed by Bender may be appropriate: ${ }^{4}$

- An absence of pain or swelling

- Disappearance of any sinus tracts

- Radiographic evidence of resolved or arrested areas of rarefaction after a post-treatment interval of 6 to 24 months

- No loss of function Given these guidelines, currently, terms like 'healed', 'healing' or 'disease' may be better expressions of treatment outcome. ${ }^{5}$

- Healed:Completely normal appearance clinically and radiologically. This also includes the appearance of a scar after periapical surgery (radiographically).

- Healing (in progress): Clinically normal tooth with reduction in size of radiolucency for a follow-up period less than 4 years.

- Disease (refractory, recurrent apical periodontitis): Clinically, symptomatic tooth regardless of radiologic appearance or presence of radiolucency (new, increased, unchanged or reduced) regardless of clinical presentation.

Recently, it has been suggested that the term 'effective' would include the categories 'healed' and 'healing' and will not result in further treatment, while the term 'ineffective' at 1 year would mean the emergence or enlargement of the periapical radiolucency and/or symptoms and signs that will require intervention. ${ }^{6}$

The advantages of using 'effective' and 'ineffective' over previous terms to describe the outcome are the following: ${ }^{6}$

- Shorten the follow-up period from 4 to 1 year, and thereby increase the recall rate and reduce the number of appointments and radiographs.

- Reduce the number of unnecessary retreatments indicated by adhering to previous definitions.

- The terms 'effective' and 'ineffective' relate directly to an indication for treatment and make clinical decisions easier and reproducible.

\section{FACTORS AFFECTING TREATMENT OUTCOME}

\section{Related to Diagnosis}

A significant factor affecting treatment outcome and one, i.e. frequently overlooked is an erroneous diagnosis. Incorrect diagnosis of pain and misinterpretation of the radiographic appearance of a bony lesion are the two common causes for a mistake in diagnosis.

- Oral pathologist Bhaskar SN has listed 38 radiolucent lesions of the jaw of which only three are of endodontic origin. Nonendodontic rarefactions that mimic a periapical lesion of endodontic origin are: odontogenic lesions, like lateral periodontal cyst, ameloblastoma, cementoma, developmental lesions like median anterior maxillary cyst, median mandibular developmental cyst, globulomaxillary cyst, oral tumors like giant cell granuloma (common in mandible), neurofibroma (mimic endodontic-periodontal lesions) and squamous cell carcinoma, physical injury like traumatic bone cyst. ${ }^{7}$

- Incorrect diagnosis of pain: The commonest nonodontogenic causes of pain that mimic pulpal and/or periapical pain are trigeminal neuralgia and maxillary sinusitis.

\section{Preoperative Condition of the Patient and the Tooth}

- Age, gender and health of the patient: Several studies have evaluated the role of the patient's age and gender on the success of endodontic treatment. They have not been able to find any correlation. ${ }^{8}$ Thus, age and gender do not influence the outcome of endodontic treatment. ${ }^{9}$ Apical periodontitis represents a balance between intraradicular infection and host defences. In the past, it was believed that host factors supposedly exerted a major influence on treatment outcome. ${ }^{10}$ However, this has not been found to be true. Only in severely immunocompromised patients and in uncontrolled diabetics has healing shown to be delayed and less predictable. Even in such cases, root canal treatment is not contraindicated as healing is still possible.

- Presence of apical periodontitis: Studies evaluating the outcome of endodontic treatment have pinpointed the presence of apical periodontitis as the single most important factor affecting healing. Teeth with irreversible pulpitis have a better prognosis than those with pulp necrosis and apical periodontitis. Sjögren et al while evaluating the long-term results of endodontic treatment concluded that the presence of preoperative periapical lesions reduced the success rate from 96 to $86 \%{ }^{3}$ Strindberg attributed this lower success rate to difficulties in the repair potential of the periapical tissues and inability to thoroughly disinfect the root canal system.

- Size of the periapical lesion: Many researchers have reported that teeth with periapical lesions lesser than $5 \mathrm{~mm}$ in diameter heal better following endodontic therapy than those with larger lesions. ${ }^{5}$ But Sjögren et al in their 8 to 10-year follow-up of 356 cases did not find this to be true. They attributed this to two possible reasons-observation periods of earlier studies were too short to re-establish normal periapical architecture and larger periapical lesions may heal by soft-tissue 
scarring which cannot be distinguished from persistent inflammation on the radiograph. ${ }^{3}$

- Condition and position of the tooth: Factors that may compromise treatment outcome include difficult access, coronal breakdown, anatomic complexities, calcifications, resorptions altering the root canal space and cracked teeth. Since these problems are more common in posterior teeth, their treatment outcome may be less predictable. Studies evaluating the healing rate for multirooted teeth give conflicting results. Earlier studies by Strindberg and Engstrom reported a higher success rate while considering each root of a posterior as a unit. The more recent Toronto study, however, reports a lower success rate for posterior teeth as they considered the tooth as a whole. ${ }^{11}$

- Nature of the canal flora: The persistence of microbial infection in the root canal system and/or the periapical area is an important cause for endodontic failure. This is true even when the treatment is technically of a very high standard. During cleaning and shaping procedures, instrumentation, irrigation and intracanal medicament are relied upon to completely eliminate microorganisms. But, studies have demonstrated that regardless of the technique or instruments used, part of the root canal space often remains untouched. Also, bacteria harbored in isthmuses, ramifications, apical deltas and dentinal tubules may sometimes be unaffected by irrigants or medicaments. However, failure will result only when these bacteria possessing pathogenicity, reach sufficient numbers and communicate with the periradicular tissue. ${ }^{12}$

If the case is poorly treated, a greater number of microbial species predominated by anaerobes similar to that found in the primary infection is likely to be found. On the other hand, well-treated cases that have failed, often show one or a few species of which E. fecalis is found in 29 to $38 \%$ of the cases. ${ }^{2}$ Yeast like microorganisms have also been isolated from the canals. ${ }^{12}$

Recently, there is considerable interest regarding the role of extraradicular infection (an established infection on the external root surface forming a biofilm and persisting in the periapical tissues) in the failure of well-treated cases. Such an infection is inaccessible to conventional endodontic disinfection procedures, and since they are in the form of a biofilm they also escape the action of the host's defences. Oral microorganisms, such as Actinomyces spp. and Propionibacterium propionicium are implicated in extraradicular infections., ${ }^{2,12}$

- Type of periapical lesion: It is seen that periapical lesions considered to be granulomas often heal following routine endodontic treatment. This is also the case with apical pocket cysts in which the cyst cavity communicates with the root canal system.
Apical true cysts in which the cystic cavity is completely enclosed in the epithelial lining and has no communication with the root canal system do not heal following nonsurgical endodontic treatment. This is because in true cysts the tissue dynamics are self-sustaining and independent of the presence or absence of irritants in the root canal system. True cysts contain large numbers of cholesterol crystals in the connective tissue around the cystic epithelial lining. These are derived from the breakdown of host cells like erythrocytes, lymphocytes, plasma cells and macrophages. Multinucleated giant cells are ineffective in removing these crystals; thus, they continue to accumulate and maintain the cyst. ${ }^{12}$

\section{Standard of Care during Endodontic Treatment}

- Apical extent of the canal preparation and obturation: The apical limit of preparation and obturation does not appear to be that critical for healing following nonsurgical endodontic treatment in teeth without apical periodontitis. In teeth with apical periodontitis, healing is optimal if the preparation and obturation is within $2 \mathrm{~mm}$ of the root apex. Over instrumentation with consequent overfilling appears to impart periapical healing to the greatest extent in teeth with apical periodontitis. ${ }^{3}$ Extrusion of infected debris into the periapical tissues rather than overextension of the obturating material is responsible for this.

- Apical width: In root canal infections, bacteria have been shown to penetrate to a depth of 150 to $250 \mu \mathrm{m}$ into the dentinal tubules. This protects them from the effect of irrigants and medicaments. Therefore, many researchers have put forth various recommendations regarding the size of the apical preparation (apical width) necessary to successfully eliminate these bacteria. However, studies have not been able to correlate the size of the apical enlargement with the treatment outcome. Excessive apical enlargement may result in iatrogenic errors like canal transportation and ledging while minimal apical preparation carries the risk of leaving residual infected material behind. Thus, a balance between these two extremes should be achieved. ${ }^{5}$

- Single versus multivisit treatment: In recent years, the consensus regarding healing following one or multivisit root canal therapy in teeth without apical periodontitis is clear. There is no difference in the healing rates. Regarding teeth associated with apical periodontitis there are many studies that emphasize the need for intracanal medication to achieve proper disinfection and a better treatment outcome. However, prolonging the treatment for more than two sessions does not provide any additional benefits. ${ }^{5,13}$ 
- Canal preparation and obturation techniques: The instrumentation technique regardless of whether hand or rotary instruments, different rotary instrument systems or degree of taper does not influence the treatment outcome. ${ }^{14}$ At present, there is insufficient data from follow-up studies to assess the effect of intracanal medication on healing potential. One study has suggested that calcium hydroxide was superior to other medicaments or no medicaments on the long-term retention of teeth. ${ }^{15}$ Studies comparing the effect of different obturation techniques suggest that the technique used for obturation does not influence the treatment outcome. ${ }^{9}$ Contemporary methods of cleaning and shaping using a crown-down approach and rotary nitinol instruments, reduce the chances of procedural errors like ledging, blockages, apical transportation, extrusion of debris, etc. Thus, if a clinician employs a scientifically accepted protocol for treatment and a three-dimensional obturation extending to within $2 \mathrm{~mm}$ of the root apex there is greater likelihood of a successful outcome. ${ }^{3}$

\section{Postoperative Factors}

The following postoperative factors have been researched: 5

- Inadequate postendodontic restoration resulting in bacterial penetration and development of apical periodontitis

- Type of restoration

- Presence or absence of posts and prevalence of apical periodontitis

The results of these studies have been conflicting. However, a definitive restoration placed as early as possible following root canal treatment is important for healing although the type of the restoration is not that critical. ${ }^{14} \mathrm{~A}$ failure rate as high as $35 \%$ has been reported by the Toronto study series due to poor postendodontic restorations. The technical quality of the coronal restoration was even more important for the periapical status than the quality of the endodontic treatment. ${ }^{16}$

After studying the technical aspects of treatment outcome, it has been reported that both the quality of the endodontic treatment and coronal restoration play important roles in obtaining an efficient seal of the root canal, even though it is suggested that the quality of the root-filling may be the most decisive parameter. ${ }^{14}$

\section{Monitoring the Treatment}

\section{Ongoing Maintenance}

Root treated teeth are often more vulnerable to caries than normal teeth as they are not sensitive or painful. Carious lesions can progress and quietly destroy the coronal tooth structure and reinfect the obturated root canal system. ${ }^{14}$

The other reasons for eventual failure are loss of integrity of the coronal restoration fracture of the crown or root development of advanced periodontal disease and traumatic occlusion. ${ }^{8}$ Therefore, careful periodic clinical and radiographic evaluation is a must.

\section{Evaluation}

Periodic radiographic evaluation should be performed to monitor post-treatment healing:

- Approximately, $90 \%$ of cases that are healing will demonstrate clear signs of improvement and 50\% would have healed completely within a year.

- If after a year if a periapical lesion appears reduced in size, it can be safely assumed that it would continue to heal and should be evaluated.

- If a lesion does not show any change radiographically, but the tooth is asymptomatic, radiographic evaluation must be carried out for 4 years. Further healing is unlikely after 4 years at which point a final judgment can be made. ${ }^{5}$

\section{CONCLUSION}

Long-term evaluation of endodontic treatment requires an exhaustive analysis of various preoperative, treatment related and postoperative factors. In general, favorable outcome of endodontic treatment in terms of healing and functionality is very good for both teeth with and without apical periodontitis. Considering the high success rate of endodontic treatment, conservative endodontic treatment should be the first choice for teeth with good restorative and periodontal prognosis. The past few decades have witnessed the emergence of newer techniques and devices that have greatly enhanced the practice of endodontics. In the light of these advances, evaluation of current practices is an important parameter in treatment outcome.

\section{REFERENCES}

1. Vire DE. Failure of endodontically treated teeth: Classification and evaluation. J Endod 1991;17(7):338-342.

2. Siqueira JF. Aetiology of root canal treatment failure: why well-treated teeth can fail. Int Endod J 2001;34(1):1-10.

3. Sjögren U, Hägglund B, Sundqvist G, Wing K. Factors affecting the long-term results of endodontic treatment. J Endod 1990;16(10):498-504.

4. Bender IB, Seltzer S, Soltanoff W. Endodontic success-a reappraisal of criteria. Oral Surg Oral Med Oral Pathol 1966; 22(6):790-802.

5. Friedman S. Prognosis of initial endodontic therapy. Endod Topics 2002;2:59-88.

6. Wu MK, Wesselink P, Shemesh $\mathrm{H}$. New terms for categorizing the outcome of root canal treatment. Int Endod J 2011;44(11): 1079-1080. 
7. Bhaskar N. Periapical lesions-types, incidence, and clinical features. JOE 1980 Nov;6(11):845-848.

8. Basmadjian-Charles CL, Farge P, Bourgeois DM, Lebrun T. Factors influencing the long-term results of endodontic treatment: a review of the literature. Int Dent J 2002 Apr;52(2): 81-86.

9. Seltzer S, Bender IB, Turken-kopf S. Factors affecting successful repair after root canal therapy. JADA 1963;67(5):651-662.

10. Takahashi K. Microbiological, pathological, inflammatory, immunological and molecular biological aspects of periradicular disease. Int Endod J 1998 Sep;31(5):311-325.

11. Farzaneh M, Abitbol S, Friedman S. Treatment outcome in endodontics: the Toronto study. Phases I and II: Orthograde retreatment. J Endod 2004;30(9):627-633.
12. Nair PN. On the causes of persistent apical periodontitis: a review. Int Endod J 2006;39(4):249-281.

13. Trope M, Delano EO, Orstavik D. Endodontic treatment of teeth with apical periodontitis: single vs multivisit treatment 1999 May;25(5):345-350.

14. Kirkevang LL, Bindsle PH. Technical aspects of treatment in relation to treatment outcome. Endo Topics 2002;2:89-102.

15. Peters LB, Wesselink PR. Periapical healing of endodontically treated teeth in one and two visits obturated in the presence or absence of detectable microorganisms. Int Endod J 2002; 35(8):660-667.

16. Ray HA, Trope M. Periapical status of endodontically treated teeth in relation to the technical quality of the root filling and the coronal restoration. Int Endod J 1995;28(1):12-18. 